TRANSACTIONS OF THE

AMERICAN MATHEMATICAL SOCIETY

Volume 350, Number 9, September 1998, Pages 3743-3765

S 0002-9947(98)01977-1

\title{
CURVE-STRAIGHTENING AND THE PALAIS-SMALE CONDITION
}

\author{
ANDERS LINNÉR
}

\begin{abstract}
This paper considers the negative gradient trajectories associated with the modified total squared curvature functional $\int k^{2}+\nu d s$. The focus is on the limiting behavior as $\nu$ tends to zero from the positive side. It is shown that when $\nu=0$ spaces of curves exist in which some trajectories converge and others diverge. In one instance the collection of critical points splits into two subsets. As $\nu$ tends to zero the critical curves in the first subset tend to the critical points present when $\nu=0$. Meanwhile, all the critical points in the second subset have lengths that tend to infinity. It is shown that this is the only way the Palais-Smale condition fails in the present context. The behavior of the second class of critical points supports the view that some of the trajectories are 'dragged' all the way to 'infinity'. When the curves are rescaled to have constant length the Euler figure eight emerges as a 'critical point at infinity'. It is discovered that a reflectional symmetry need not be preserved along the trajectories. There are examples where the length of the curves along the same trajectory is not a monotone function of the flow-time. It is shown how to determine the elliptic modulus of the critical curves in all the standard cases. The modulus $p$ must satisfy $2 E(p) / K(p)=1 \pm|g| / \widetilde{L}$ when the space is limited to curves of fixed length $\widetilde{L}$ and the endpoints are separated by the vector $g$.
\end{abstract}

\section{INTRODUCTION}

1.1 The total squared curvature. Let $\gamma$ be a smooth curve in the Euclidean plane and let $k$ be its signed curvature. The quantity $F(\gamma)=\int_{\gamma} k^{2} d s$ is known as the total squared curvature. $F(\gamma)$ has a physical interpretation as the 'elastic' energy stored in a thin rod shaped as $\gamma$. It is possible to extend the notion of curvature to curves in any Riemannian manifolds. In this case the curvature is often referred to as the geodesic curvature. A geodesic is a curve with identically zero curvature. Geodesics are global minimizers of the total squared curvature.

1.2 Closed geodesics. In order to have zero curvature a curve must satisfy the so-called 'geodesic equation'. Non-trivial periodic solutions of the geodesic equation are called closed geodesics. Note that both the curve and its tangent curve must be smooth and periodic. There is of course no guarantee that there exists a closed geodesic through a given point with a given tangent direction. The need to 'search' for correct initial data is one motivation behind the introduction of various 'energy'

Received by the editors July 17, 1995 and, in revised form, November 5, 1996.

1991 Mathematics Subject Classification. Primary 58F25; Secondary 58E10, 53C21.

Key words and phrases. Curve-straightening, Palais-Smale condition, gradient trajectories.

(C) 1998 American Mathematical Society 
reducing flows. These flows by definition take place in a space of closed curves, so the boundary conditions are automatically satisfied.

1.3 Gradient flows. An obvious candidate for an energy reducing flow is the negative gradient flow associated with the length functional $L(\gamma)=\int_{\gamma} d s=\int_{0}^{1}\left|\gamma^{\prime}(t)\right| d t$. For technical reasons it turns out that a better quantity is given by the 'total kinetic' energy $E(\gamma)=\int_{0}^{1}\left|\gamma^{\prime}(t)\right|^{2} d t$. With $E$ in mind Palais [10] and Klingenberg [2] showed how spaces of closed curves can be a given the structure of a Hilbert manifold where the notions of gradients, trajectories and flows are available.

Note that $E$ is defined and globally minimized on trivial curves (i.e., constant curves with the image a single point.) It follows that a closed geodesic in a trivial homotopy class it is not expected to be an 'attractor' of the negative gradient vector field associated with $E$.

1.4 The curve straightening flow. Things are improved if $E$ is replaced by $F$. A shrinking 'circle' has ever increasing total squared curvature, so the trivial curves are avoided. It is tempting to restrict $F$ to the curves of constant speed, since this simplifies the curvature formula a great deal. This kind of restriction does not fit the Palais-Klingenberg framework. There is however a related Hilbert manifold where the analysis of $F$ is carried out, see [4] and [5]. In this setting the flow along the negative gradient trajectories of $F$ is what is known as the curve straightening flow.

1.5 Existence of geodesics in surfaces with trivial homotopy. Imagine a compact surface diffeomorphic to a sphere. A good example is something like an asymmetrical egg. It has recently been established that there exist infinitely many 'geometrically distinct' (i.e., 'visibly' different) closed geodesics in each such surface; see [1]. The interest in the curve straightening flow is fueled by the prospect of numerical implementations which would generate closed geodesics.

1.6 The Palais-Smale condition and convergence of the trajectories. Let $\Lambda: X \rightarrow[0, \infty)$ be a smooth nonlinear functional on a complete Hilbert manifold $X$. It is important that $\Lambda$ is bounded from below. Proposition 3.1 shows that the negative gradient trajectories of $\Lambda$ extend indefinitely. As the flow-time increases without bounds the gradient of $\Lambda$ tends to zero. Let $x_{n}$ be any sequence in $X$ such that $\Lambda\left(x_{n}\right)$ is bounded and $\left\|\nabla \Lambda\left(x_{n}\right)\right\| \rightarrow 0$. The Palais-Smale condition is said to be satisfied if it is always true that $x_{n}$ has a convergent subsequence. In the context of curve straightening the P-S condition is known to be satisfied when the domain consists of curves of fixed length, or if $F$ is modified by adding a term $\nu L$ where $\nu>0$. It follows that in both cases all the trajectories converge to critical points. Another consequence is the existence of global minimizers in the two cases.

1.7 Examples where the Palais-Smale condition fails. From Section 1.6 it is seen that, to have any opportunity to violate the Palais-Smale condition, it is necessary that the length of the curves is unconstrained and that $\nu=0$. The P-S condition is not satisfied for closed curves in the standard round sphere. To see this let $\gamma_{n}$ be the curve which wraps around the equator $n$ times at a constant speed. Each $\gamma_{n}$ is a geodesic, so the total squared curvature is zero and the gradient is also zero. This sequence of curves does not converge. Note that this is not an example of a negative gradient trajectory which does not converge. It is not known if any of the trajectories diverge in this case. 
Another example is given by the closed curves in the Euclidean plane. In this case there is no global minimizer. To see this, consider circles of ever increasing radius. The total squared curvature tends to zero, but there is no closed geodesic in the Euclidean plane.

1.8 Motivation. The purpose of this paper is to present results that shed light on the behavior of the trajectories as $\nu$ tends to zero from the positive side. Keep in mind that all geodesics are global minimizers only if $\nu=0$. It is a significant open problem to determine if all the trajectories converge when $\nu=0$. If there are divergent trajectories it is of interest to know how this can be, especially considering the absence of divergent trajectories when $\nu>0$.

1.9 Non-closed curves in the Euclidean plane. The space of non-closed curves in the Euclidean plane serves as the primary example in this paper. There is a global minimum given by the straight line segment between the two endpoints. Unlike the case of closed curves, this is consistent with a satisfied P-S condition. Interestingly, there is also an infinite but countable collection of non-geodesic critical points. This collection does not yield a sequence which violates the P-S condition, because there is no infinite subcollection on which $F$ is bounded.

1.10 The main results. In the space of non-closed curves in the Euclidean plane it is shown that when $\nu=0$ some trajectories converge and others diverge. This happens despite the fact that when $\nu$ is positive all trajectories converge. The underlying source of this behavior is discovered.

When $\nu$ is positive the collection of critical points is separated into two subcollections. As $\nu \rightarrow 0^{+}$the curves in the first sub-collection converge to the critical points present when $\nu$ is zero. Curves in the second sub-collection have lengths which tend to infinity as $\nu \rightarrow 0^{+}$. In this paper the second kind of critical points are said to 'migrate' to infinity. If the curves of ever increasing length are 'normalized' to have a fixed length, then the Euler figure eight or half of it (and multiple covers) emerge as 'critical points at infinity'.

Several examples are given to illustrate this. These examples suggest the following picture when the space of curves is viewed as a closed unbounded infinitedimensional Hilbert manifold. When $\nu>0$ each critical point attracts some trajectories. As $\nu$ is decreased towards zero some of the critical points migrate to infinity and in the process 'drag' some of the trajectories to infinity. When $\nu=0$ there are invisible critical points at 'infinity' that attract trajectories.

1.11 Some additional results. The author's work [9] is here extended to include the case of non-zero $\nu$. When the Euler-Lagrange system is reduced it yields some strikingly compact formulas which determine the Jacobi elliptic modulus. The relationship between the parameter $\nu$ and the length constrained Lagrange multiplier is clarified. Proposition 3.2 implies that the P-S condition is satisfied when the curves have bounded length. A series of numerical simulations are presented which illustrate the negative gradient flow. The case of planar closed curves of fixed length is illustrated in [6] and [7]. In [6] it is shown that certain rotational and reflectional symmetries are preserved. By contrast it is here shown that for non-closed curves the prevalent behavior is to break reflectional symmetry. There may be a different choice of Riemannian metric in the space of curves where reflectional symmetry is preserved under curve straightening. 
It is shown in [9] that there are very long curves with arbitrary small total squared curvature. There are trajectories where the lengths of the curves are monotonically increasing. There are also trajectories converging to the straight line between the endpoints where the lengths of the curves are monotonically decreasing. What is somewhat surprising (if not annoying) is the existence of trajectories on which the curves temporarily get longer before they eventually get shorter. The total squared curvature is of course always monotonically decreasing. This new fact is yet another example of the many differences between curve straightening and the Gage-Hamilton-Grayson curve-shortening flow.

1.12 The principal of constraint removal. The spaces of curves associated with the various types of constraints are all represented as submanifolds of a single Hilbert manifold. It is very useful to solve a variational problem in the presence of all potential constraints. A case corresponding to a subset of all the potential constraints utilizes the same symbolic relationships to characterize critical points as the general case. Removing a constraint simply involves setting each multiplier associated with the constraint equal to zero, and changing the unconstrained quantity to an unknown. This preserves the number of equations as well as the number of unknowns. The principle asserts that when the general set of symbolic relationships is reinterpreted in this way, the result is the correct characterization of critical points in the special case.

1.13 Open problems for closed curves when $\nu=0$. It is not known if all the curve straightening trajectories converge in the space of closed curves in the standard round sphere. In the hyperbolic plane the P-S condition is not satisfied when the rotation number is 2 or higher. The reason is that there is no global minimizer (the case 3 or higher is in [3] and the case 2 is in [12]). When the rotation number is 1 it is not known if the P-S condition is satisfied. The work by Steinberg has ruled out the existence of migrating critical points in this case. It is not known, in any space of curves, if the absence of migrating critical points forces convergence of the curve straightening trajectories.

1.14 Organization. The paper is organized as follows:

1. Introduction.

2. Existence of critical points.

3. Behavior along the trajectories.

\section{EXISTENCE OF CRITICAL POINTS}

\subsection{The setup.}

2.1.1 Indicatrices. Consider curves $\gamma$ in the Euclidean plane. Let $C$ denote the set of complex numbers and let $I=[0,1]$. Let $\gamma: I \longrightarrow C$ be a continuously differentiable regular curve of length $L$ which is parameterized so that $\left|\gamma^{\prime}(s)\right|=L$. Associated with $\gamma$ is its real valued continuous tangent indicatrix $\theta: I \longrightarrow R$ defined by $\gamma^{\prime}(s)=L e^{i \theta(s)}$. Let $g \in C$ be given and possibly zero. Note that $\gamma$ has endpoints separated by $g$ whenever $L \int_{I} e^{i \theta}=g$. Suppose $\theta_{0}$ and $\theta_{1}$ are given real numbers. The assumptions $\theta(0)=\theta_{0}$ and $\theta(1)=\theta_{1}$ correspond to prescribing the tangent directions of $\gamma$ at the endpoints. With $g=0$ and $\theta_{1}-\theta_{0}=n \cdot 2 \pi$ the curves are $C^{1}$-closed and $n$ is the rotation number of $\gamma$. 
2.1.2 The choice of domain. The signed curvature $k$ of $\gamma$ is given by $k=\dot{\theta} / L$. Our analysis concerns the modified total squared curvature functional $J^{\nu}(\theta, L)=$ $\left(\frac{1}{2 L} \int_{I} \dot{\theta}^{2}\right)+\nu L$, where $\nu$ is a real valued parameter. The largest possible domain for $J^{\nu}$ is given by $H \times R^{+}$, where $H$ is the Sobolev space of absolutely continuous functions with Lebesgue square integrable derivatives, and $R^{+}$is the set of positive reals. We identify the tangent spaces with $H \times R$ and use the inner product

$$
\left\langle\left(v_{\theta}, v_{L}\right),\left(w_{\theta}, v_{L}\right)\right\rangle=v_{\theta}(0) w_{\theta}(0)+\int_{I} \dot{v}_{\theta} \dot{w}_{\theta}+v_{L} w_{L} .
$$

2.1.3 Constraints. The space $H \times R^{+}$represents all curves in the plane. The analysis concerns three different types of constraints. Each individual constraint 'generates' a closed submanifold of $H \times R^{+}$. If two or more constraints are present, one must check that the associated submanifolds intersect transversally in order to conclude that the intersection is a manifold. Curves that are constrained to have endpoints separated by $g$ constitute a corresponding submanifold denoted by $\Omega$. If the curves are required to have some fixed length $\widetilde{L}$, write $\Omega_{\widetilde{L}}$. It the angle is fixed at a parameter $\widetilde{s}$, write $\Omega_{\theta_{\tilde{s}}}$.

Let the following functionals be defined on $H \times R^{+}: \Lambda_{g}(\theta, L)=L \int_{I} e^{i \theta}-g$, $\Lambda_{\widetilde{L}}(\theta, L)=L-\widetilde{L}$, and $\Lambda_{\theta}(\theta, L)=\theta(\widetilde{s})-\theta_{\widetilde{s}}$. The submanifolds $\Omega, \Omega_{\widetilde{L}}$ and $\Omega_{\theta_{\tilde{s}}}$ are given as inverse images of 0 using these three functionals.

To be convinced that $\Omega, \Omega_{\widetilde{L}}$ and $\Omega_{\theta_{\tilde{s}}}$ are submanifolds, compute the directional derivatives of the three functionals and show that they are onto. Since $H \times R^{+}$ is complete 'locally', the implicit function theorem is applicable. Let $\left(v_{\theta}, v_{L}\right)$ be a tangent vector; then $D \Lambda_{g}\left(v_{\theta}, v_{L}\right)=v_{L} \int_{I} e^{i \theta}+i L\left\{v_{\theta}(1) \int_{I} e^{i \theta}-\int_{i} \dot{v}_{\theta} \int_{0}^{s} e^{i \theta}\right\}$, $D \Lambda_{\widetilde{L}}\left(v_{\theta}, v_{L}\right)=v_{L}$, and $D \Lambda_{\theta}\left(v_{\theta}, v_{L}\right)=v_{\theta}(\widetilde{s})$. It is not difficult to check that these maps are onto. The only time things are not straightforward is when $g=0$; see $[8]$.

2.1.4 Gradient. Recall that if $F: X \longrightarrow R$ is a functional on a Riemannian (Hilbert) manifold then the gradient $\nabla F(x)$ is defined using the Riesz representation theorem. For each fixed $x \in X$ it is required that $D F(x) v=\langle\nabla F(x), v\rangle_{x}$ for all tangent vectors $v \in T_{x} X$. The directional derivative of $J^{\nu}$ in the direction $v=\left(v_{\theta}, v_{L}\right)$ is given by

$$
D J^{\nu}(\theta, L) v=\frac{1}{L} \int_{I} \dot{\theta} \dot{v}_{\theta}-\frac{v_{L}}{2 L^{2}} \int_{I} \dot{\theta}^{2}+\nu v_{L} .
$$

It is easy to check that $\nabla J^{\nu}(\theta, L)=\left(\frac{1}{L}(\theta(s)-\theta(0)),-\frac{1}{2 L^{2}} \int_{I} \dot{\theta}^{2}+\nu\right)$.

In the presence of constraints, an element $(\theta, L)$ is said to be a critical point whenever the tangential projection of $\nabla J^{\nu}(\theta, L)$ vanishes. The tangential projection is computed by subtracting the normal component of $\nabla J^{\nu}(\theta, L)$ from $\nabla J^{\nu}(\theta, L)$. Note that the normal spaces associated with the constraints considered in this paper are finite dimensional. The following is a standard result in Hilbert space theory. Every continuous linear map which is onto has a transpose which is onto the space normal to the kernel of the linear map. In our case the manifolds are given as level sets, so the kernel is equal to the tangent space.

2.1.5 Span of the normal space. In the complex plane $C$ use the real inner product $\langle v, w\rangle_{C}=\operatorname{Re}(v \bar{w})$. Given a complex number $\lambda$, determine $D \Lambda_{g}^{T}$ so that

$$
\left\langle D \Lambda_{g}\left(v_{\theta}, v_{L}\right), \lambda\right\rangle_{C}=\left\langle\left(v_{\theta}, v_{L}\right), D \Lambda_{g}^{T}(\lambda)\right\rangle .
$$


The formulas are derived in [8] and they also appear later in Proposition 3.2. The normal spaces of $\Omega_{L}, \Omega_{\theta_{0}}$ and $\Omega_{\theta_{1}}$ are one-dimensional and are spanned by $\nabla \Lambda_{\tilde{L}}=$ $(0,1), \nabla \Lambda_{\theta_{0}}=(1,0)$ and $\nabla \Lambda_{\theta_{1}}=(1+s, 0)$ respectively.

When several of the constraints are imposed at the same time the corresponding submanifold is the intersection of each individual submanifold. In the context covered by this paper the various submanifolds intersect transversally; see [8]. It follows that the intersection is also a manifold. It is important to determine every single critical point. Once this is done, the analysis of the behavior of the negative gradient trajectories can proceed.

2.2 The critical points. Recall that the solutions of the fundamental differential equation are given in terms of Jacobi elliptic functions as follows.

Proposition 2.1. Given any real numbers $\varphi_{0}, \dot{\varphi}_{0}$ and $c$, the unique solution of the initial value problem

$$
\ddot{\varphi}(s)+\frac{\varphi^{3}(s)}{2}+c \varphi(s)=0, \quad \varphi(\widetilde{s})=\varphi_{0}, \dot{\varphi}(\widetilde{s})=\dot{\varphi}_{0}
$$

is given by either

I. $\varphi(s)=A c n[\alpha s+\beta]$ of modulus $p \in[0,1]$ with $A c n \beta=\varphi_{0},-A \alpha s n \beta d n \beta=\dot{\varphi}_{0}$ and $A^{2}=4 p^{2} \alpha^{2}, c=\alpha^{2}\left(1-2 p^{2}\right)$, or

II. $\varphi(s)=\operatorname{Adn}[\alpha s+\beta]$ of modulus $p \in[0,1]$ with $\operatorname{Adn} \beta=\varphi_{0},-A \alpha p^{2} \operatorname{sn} \beta c n \beta=$ $\dot{\varphi}_{0}$ and $A^{2}=4 \alpha^{2}, c=\alpha^{2}\left(p^{2}-2\right)$.

If $4 \dot{\varphi}_{0}^{2}+\left(\varphi_{0}^{2}+4 c\right) \varphi_{0}^{2} \geq 0$ then the solution is given by case I; otherwise the solution is given by case II.

The proof is given in [9].

In order to use the principle of constraint removal mentioned in section 1.12, impose all constraints under consideration. Specifically, assume the curves have fixed length $\widetilde{L}$ with endpoints whose difference is given by the complex number $g$ with $|g|<\widetilde{L}$ and tangent directions with angles $\theta_{0}, \theta_{1}$ at the endpoints.

Theorem 2.2. The point $(\theta, L) \in H \times R^{+}$is a critical point of $J^{\nu}(\theta, L)=\left(\frac{1}{2 L} \int_{I} \dot{\theta}^{2}\right)$ $+\nu L$ subject to $L=\widetilde{L}, \theta(0)=\theta_{0}, \theta(1)=\theta_{1}$ and $L \int_{I} e^{i \theta}=g$, if and only if $\dot{\theta}(s)=A f[\alpha s+\beta]$, where $f$ is the Jacobi elliptic cn or dn of modulus $p$. We have cn if $4\left\langle i e^{i \theta_{0}}, \lambda\right\rangle^{2}+\left(\mu_{0}^{2}-4(\nu+\mu)\right) \mu_{0}^{2} \geq 0$ and $d n$ otherwise. In the case of $c n$ we have $p^{2}=\frac{1}{2}\left(1+\frac{\nu+\mu}{|\lambda|}\right)$ and $A^{2}=4 p^{2} \alpha^{2}$. In the dn case, $p^{2}=2-\frac{\nu+\mu}{|\lambda|}$ and $A^{2}=4 \alpha^{2}$. The unknown $\lambda, \alpha, \beta, p, \mu_{0}$ and $\mu_{1}$ must solve the following system, where $f$ stands for $\mathrm{cn}$ or $d n, P=p$ in the case of $\mathrm{cn}$ and $P=1$ in the case of $d n$ :

$$
\begin{gathered}
|g||\lambda| \sin [\operatorname{Arg}(g)-\operatorname{Arg}(\lambda)]=\mu_{0}+\mu_{1}, \\
\pm 2 \alpha P f[\beta]=\widetilde{L} \mu_{0}, \\
\pm 2 \alpha P f[\alpha+\beta]=-\widetilde{L} \mu_{1}, \\
\alpha^{2} p^{2}=\widetilde{L}^{2} P^{2}|\lambda|, \\
\pm 2 \sin ^{-1}(\operatorname{Psn}[\beta])+\operatorname{Arg}(\lambda)=\theta_{0},
\end{gathered}
$$




$$
\begin{gathered}
\pm 2 \sin ^{-1}(P \sin [\alpha+\beta])+\operatorname{Arg}(\lambda)=\theta_{1}, \\
2 \widetilde{L}\{E[\alpha+\beta]-E[\beta]\}-\widetilde{L} \alpha=\alpha|g| \cos [\operatorname{Arg}(g)-\operatorname{Arg}(\lambda)], \quad \text { cn-case, } \\
2 \widetilde{L}\{E[\alpha+\beta]-E[\beta]\}-\widetilde{L} \alpha\left(p^{2}-2\right)=\alpha p^{2}|g| \cos [\operatorname{Arg}(g)-\operatorname{Arg}(\lambda)], \quad d n \text {-case. }
\end{gathered}
$$

Proof. Assume that $(\theta, L) \in H \times R^{+}$is critical and that it satisfies the constraints

$$
\begin{gathered}
L=\widetilde{L}, \\
\theta(0)=\theta_{0}, \\
\theta(1)=\theta_{1}, \\
L \int_{I} e^{i \theta}=g .
\end{gathered}
$$

There must be numbers $\mu, \mu_{0}, \mu_{1}$ and $\lambda$ such that

$$
\frac{1}{\widetilde{L}}\left(\theta(s)-\theta_{0}\right)+\left\langle-i \widetilde{L} \int_{0}^{s} \int_{0}^{u} e^{i \theta}+i(1+s) g, \lambda\right\rangle+\mu_{0}+\mu_{1}(1+s)=0
$$

and

$$
-\frac{1}{\widetilde{L}^{2}} \int_{I} \dot{\theta}^{2}+\nu+\left\langle\frac{g}{\widetilde{L}}, \lambda\right\rangle+\mu=0
$$

Note how the parameter $\nu$ and the multiplier $\mu$ take on similar roles in this condition. If we let $s=0$ in (5), and use (2), we get

$$
\langle i g, \lambda\rangle+\mu_{0}+\mu_{1}=0,
$$

so the failure of the multiplier $\lambda$ to be perpendicular to $g$ is due to the presence of constraints on the tangent direction. If the curve is closed, then $g=0$ and (7) implies that $\mu_{0}=-\mu_{1}$. Combining (5) and (7), we get

$$
\frac{1}{\widetilde{L}}\left(\theta(s)-\theta_{0}\right)=\left\langle i \widetilde{L} \int_{0}^{s} \int_{0}^{u} e^{i \theta}, \lambda\right\rangle+\mu_{0} s
$$

The expression to the right of the equality is twice differentiable in $s$, so if there is a critical $\theta$ it must be twice differentiable, but then the expression is really four times differentiable and so on and so forth. The conclusion is that if $\theta$ is critical it must be $C^{\infty}$. If (8) is differentiated twice, we get

$$
\begin{gathered}
\frac{\dot{\theta}(s)}{\widetilde{L}}=\left\langle i \widetilde{L} \int_{0}^{s} e^{i \theta}, \lambda\right\rangle+\mu_{0}, \\
\frac{\ddot{\theta}(s)}{\widetilde{L}}=\left\langle i \widetilde{L} e^{i \theta(s)}, \lambda\right\rangle .
\end{gathered}
$$

Equation (10) has $\dot{\theta}$ as an integrating factor, so that

$$
\frac{\dot{\theta}^{2}(s)}{2 \widetilde{L}}=\left\langle\widetilde{L} e^{i \theta(s)}, \lambda\right\rangle+C
$$

To determine $C$, the constant of integration, integrate (11) from 0 to 1 and use (6) to conclude that $C=\widetilde{L}(\nu+\mu)$. If (10) is differentiated and (11) is used, we get

$$
\dddot{\theta}(s)+\frac{\dot{\theta}^{3}(s)}{2}-\widetilde{L}^{2}(\nu+\mu) \dot{\theta}=0 .
$$


From (9) we get the boundary values $\dot{\theta}(0)=\widetilde{L} \mu_{0}$ and $\dot{\theta}(1)=-\widetilde{L} \mu_{1}$. A comparison with Proposition 2.1 shows that the solutions can only be the Jacobi elliptic $c n$ or $d n$ functions and the deciding inequality takes the form

$$
4\left\langle i e^{i \theta_{0}}, \lambda\right\rangle^{2}+\left(\mu_{0}^{2}-4(\nu+\mu)\right) \mu_{0}^{2} \geq 0
$$

as claimed. The only possibilities are

$$
\dot{\theta}(s)=A c n[\alpha s+\beta] \quad \text { and } \quad \dot{\theta}(s)=A d n[\alpha s+\beta] .
$$

In the case of $c n$ solutions we have $p^{2}=\frac{1}{2}\left(1+\frac{\nu+\mu}{|\lambda|}\right)$ and $A^{2}=4 p^{2} \alpha^{2}$. In the $d n$ case, $p^{2}=2-\frac{\nu+\mu}{|\lambda|}$ and $A^{2}=4 \alpha^{2}$. We are now in a position to apply the general theorem 4.1 of [9] with the simplification that $n=0$. Note that the proof of theorem 4.1 in [9] does not include the $\nu \widetilde{L}$ term.

2.3. Reductions. It is of no interest to have both $\nu>0$ and a length constraint at the same time. The two cases $\nu>0$ (with no length constraint), and $\nu=0$ (with length constraint) are to be analyzed. We begin with the latter.

2.3.1 Length constrained and $\nu=0$. The objective is to reduce the number of unknowns and equations. As it turns out, the dimension of the system is equal to the number of angular constraints plus one. If the tangent directions at the endpoints are free then there is only one equation left to be solved. All other quantities are determined subsequently.

Suppose the tangent directions are given at both endpoints. Then it suffices to solve the following system:

$$
\begin{gathered}
\pm\{c n[\alpha+\beta]-c n[\alpha]\}=-\frac{\alpha}{\widetilde{L}} \sin \left(\theta_{0} \mp 2 \sin ^{-1}(\operatorname{ssn}[\beta])-\operatorname{Arg}(g)\right), \\
\pm 2\left\{\sin ^{-1}(p s n[\alpha+\beta])-\sin ^{-1}(p \operatorname{sn}[\beta])\right\}=\theta_{1}-\theta_{0}, \\
\pm \frac{2 p\{c n[\alpha+\beta]-c n[\alpha]\}}{2\{E[\alpha+\beta]-E[\beta]\}-\alpha}=-\tan \left(\theta_{0} \mp 2 \sin ^{-1}(p s n[\beta])-\operatorname{Arg}(g)\right) .
\end{gathered}
$$

The unknowns are $\alpha, \beta$ and the modulus $p$. There is a similar system in the $d n$ case and the choice between $c n$ and $d n$ is governed by the inequality of Theorem 2.2 . Now suppose the tangent directions are free at the endpoints. Using the principle of constraint removal, let $\mu_{0}=\mu_{1}=0$. Note that the inequality of Theorem 2.2 is satisfied, so only $c n$ solutions are possible. Since $c n[\beta]=0$, assume that $\beta=-K$, so that $c n[\alpha-K]$ implies $\alpha=r \cdot 2 K$ for some integer $r$. The periodicity of the Jacobi elliptic integrals $E$ and $K$ reveal that $2 r E[K]=(2 r-1) E[K]+E[K]=$ $E[r \cdot 2 K-K]-E[-K]=r K\left(1 \pm \frac{|g|}{\widetilde{L}}\right)$. It follows that the modulus $p$ must be such that $\frac{2 E[K]}{K}=1 \pm \frac{|g|}{\tilde{L}}$. The derivative formulas $d K / d p=p \int_{0}^{K} s n^{2} x / d n^{2} x d x$ and $d E / d p=-p \int_{0}^{K} s n^{2} x d x$ show that $d K / d p>0$ and $d E / d p<0$ for $0<p<1$, so $2 E[K] / K$ is monotonically decreasing as a function of $p$, which implies that for every nonzero $g$ there are exactly two possible moduli. 


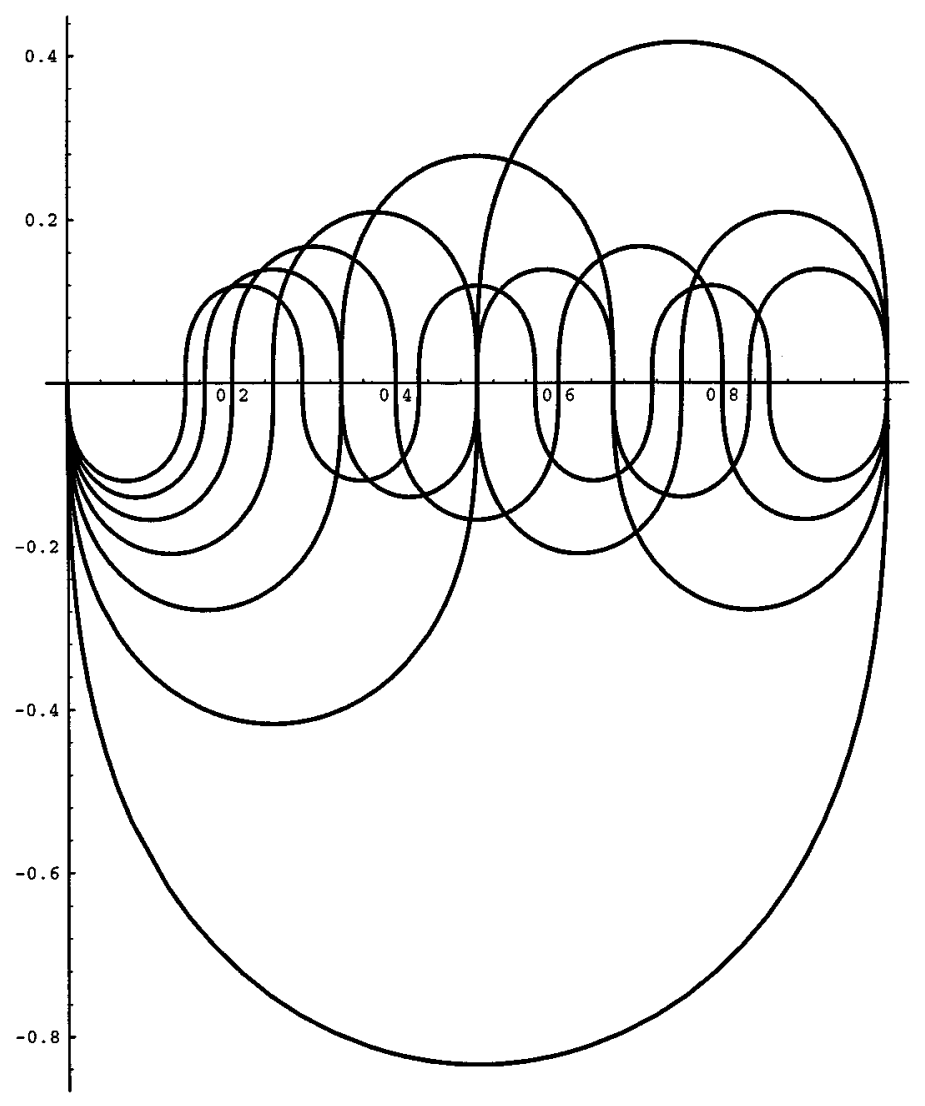

FIGURE 1

2.3.2 No length constraint. The case $\nu=0$ is completely resolved in [8] and can also be recovered from Theorem 2.2 above. The most significant simplifications result from the fact that $\mu=0$, so that the inequality of Theorem 2.2 is satisfied. Only $c n$ solutions occur, and the modulus is always $p^{2}=\frac{1}{2}$. As a consequence the resulting system contains two unknown when the directions are given at the endpoints. If the tangent directions are free then all critical points can be given explicitly. For each nonzero integer $r$ there is a pair of critical points. We will not distinguish between the two, since they only differ by a reflection. From now on we talk only about the critical point for each $r$. All of these critical points have the same length; see Figure 1, where the first seven are listed, i.e., $r=1, \cdots, 7$. The only other critical point is the global minimum, which is the straight line segment and is associated with $r=0$.

The case $\nu>0$ yields the same set of equations with the following important difference. The length $\widetilde{L}$ is not given and should be replaced by $L$. This gives us one more unknown but there is also one more equation, namely $p^{2}=\frac{1}{2}\left(1+\frac{\nu L^{2}}{\alpha^{2}}\right)$. We use this equation to solve for $L$ and substitute in the remaining three equations. This gives us a new, slightly more complicated system, of three equations where $\alpha, \beta$ and $p$ are the unknowns. Note that both $c n$ and $d n$ are possible solutions. 


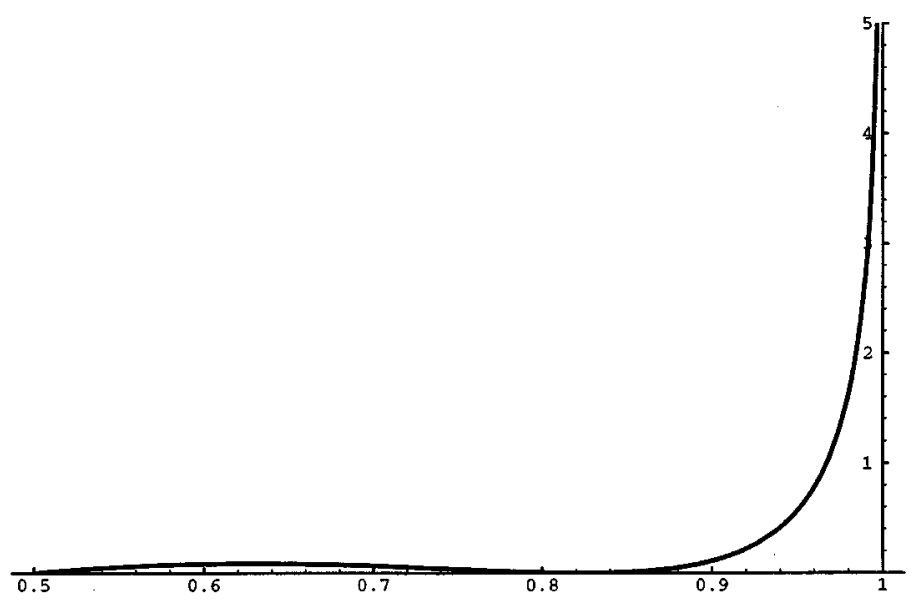

FIGURE 2

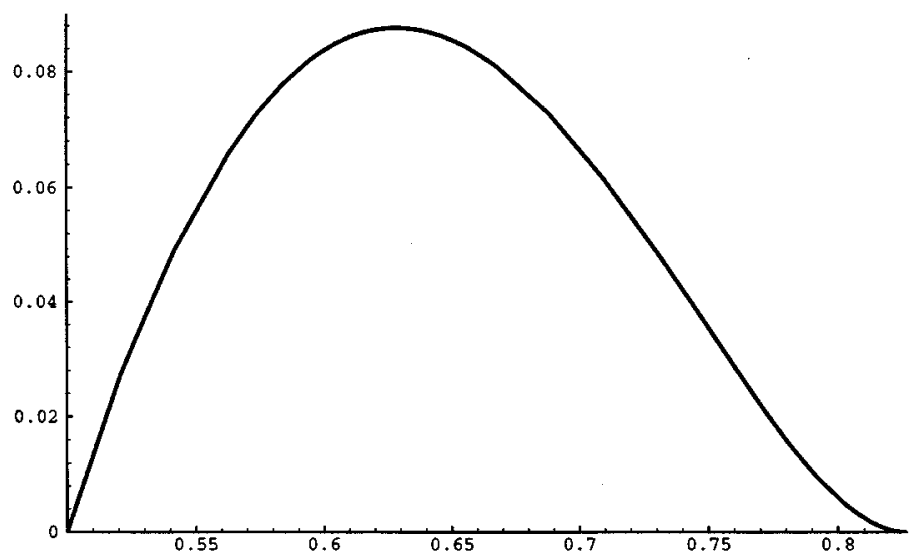

FiguRE 3

2.3.3 Free endpoint directions. To reach our goal we finally consider the special case with unconstrained tangent directions at the endpoints. Since $\mu_{0}=0$ the inequality in Theorem 2.2 is satisfied, and therefore only the $\mathrm{cn}$ solution is possible. Mimicking the length constrained case, we again get $2 E[K] / K=1 \pm|g| / L$, but here $L$ is not known. This distinction is not necessary if $g=0$, i.e., the case of closed curves. If $g \neq 0$, solve for $L$ and get $L=\frac{K}{|2 E[K]-K|}|g|$. Since $p^{2}=\frac{1}{2}\left(1+\frac{\nu L^{2}}{(2 r K)^{2}}\right)$, it possible to eliminate $L$ and get $\left(2 p^{2}-1\right)(2 E[K]-K)^{2}=\frac{\nu}{4 r^{2}}|g|^{2}$. The right-hand side of this equation is a function $f\left(p^{2}\right)$ of the modulus which satisfies $f(0)=-\frac{\pi^{2}}{4}, f(1 / 2)=0$ and $f(1)=+\infty$. The interest is in nonnegative $\nu$, so restrict to $p^{2} \geq 1 / 2$. The left-hand side is a function $g(\nu, r)$. Let $p_{0}$ be the modulus such that $2 E=K$; then $f\left(p_{0}^{2}\right)=0\left(p_{0}^{2} \approx 0.826115\right)$. (It is interesting to note that $2 E=K$ is satisfied for length constrained curves in the case of closed curves.) As can be seen in Figures 2 and 3 , there is a local maximum $p_{m}^{2} \approx 0.628168$ with $f\left(p_{m}^{2}\right)=m \approx 0.0876342$. 
2.3.4 Fixed $\nu$. For fixed $\nu$ it is seen that for sufficiently large 'modes' $r$ ( $r$ essentially corresponds to the number of inflection points) the value of $g(\nu, r)$ is lower than $m$. For such $r$ 's there are therefore two sub-collections of critical points. As $\nu$ is decreased, lower modes are admitted into this splitting. Note how the kind corresponding to larger modulus $p$ has lengths that tend to infinity. If $\nu$ is sufficiently small then all modes are split. The length formulas also show that $p \geq p_{0}$ is not admitted, since $L$ is either undefined or negative. The only other critical point is the geodesic between the endpoints. The geodesic corresponds to $r=0$, and is not part of the current analysis.

2.3.5 Variable $\nu$. For fixed $r$ observe that as $\nu$ is made smaller there is some instance $\nu_{m}$ when $g\left(\nu_{m}, r\right)=m$. As this takes place a 'new' critical point appears (as mentioned above one really gets two critical points, but they differ by a reflection only). If $\nu$ is made even smaller, this critical point 'bifurcates' into two distinct

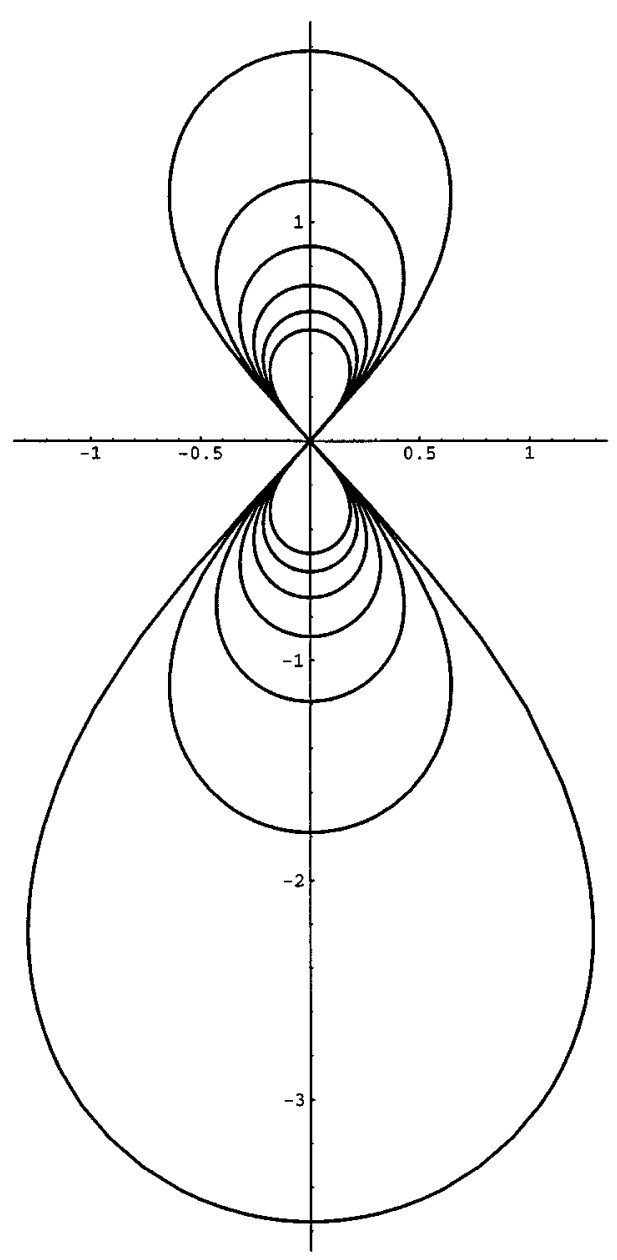

Figure 4 
critical points with different elliptic moduli. As $\nu$ tends to zero one critical point 'migrates' to infinity in that the length of the corresponding curve becomes arbitrarily large. Their moduli also tend to $p_{0}$, i.e., the modulus required for closed length constrained curves. From this one expects that in the case of $\nu=0$ curves whose lengths tend to infinity ultimately resemble one loop (or more) of the classical 'Euler figure eight'; see Figure 4, where the first 7 modes are shown with fixed length. As mentioned in the introduction, this offers an example of an attracting critical point at infinity. Finally, by examining the explicit formulas it is seen that the remaining critical points approach the corresponding $r$-kind critical points present in the unconstrained length case with $\nu=0$.

\section{BEHAVIOR ALONG THE TRAJECTORIES}

One of the most striking properties of the curve-straightening flow in the space of nonclosed curves is its 'desire' to break symmetry. To illustrate this by an explicit example, consider a semi-circle as initial curve. This curve converges to the global minimizing straight line segment between its endpoints; see Figure 5. Examine the curve $\mathrm{d} 3$ at 0.2 and 0.8 and note the slight 'discrepancy'. The obvious suspect is of course the numerical inaccuracy in the implementation. However, as seen below, the gradient does not preserve the reflectional symmetry.

$\mathrm{Y}$

\section{Graph}

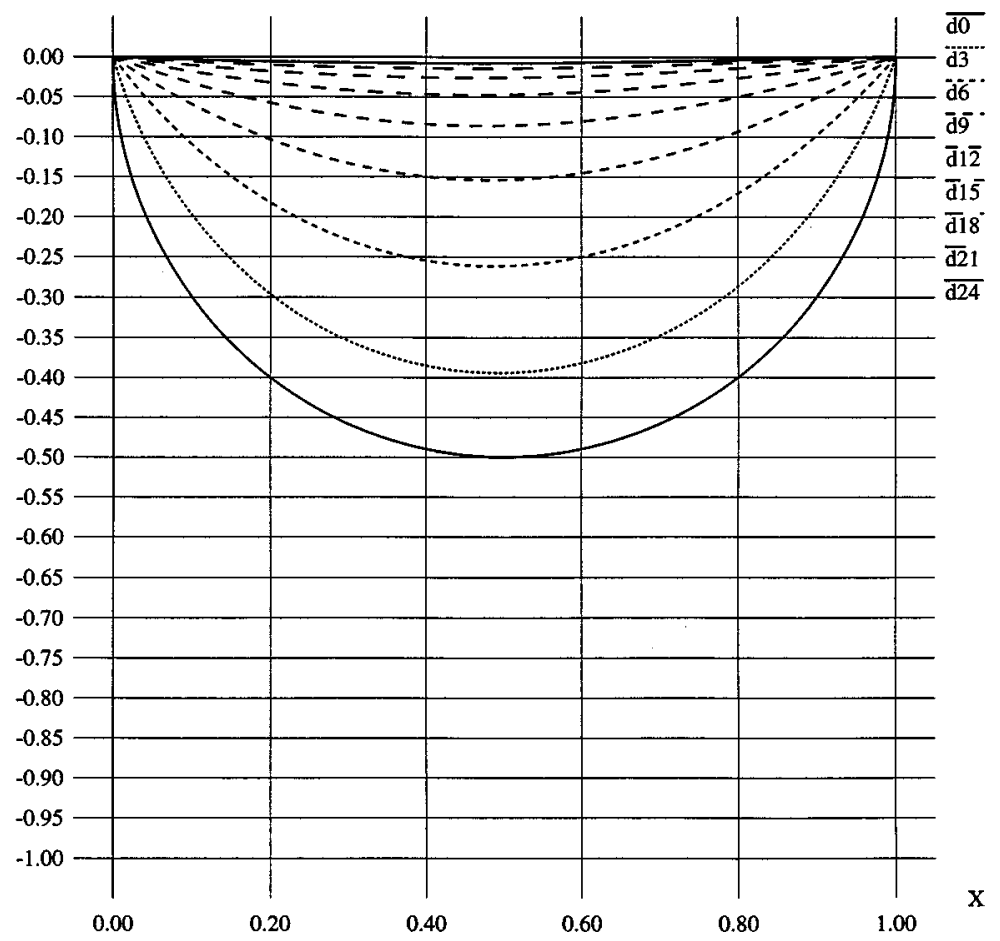

Figure 5 
3.1 Flow equations. To facilitate this particular computation introduce $G_{a}$ : $H \times R^{+} \longrightarrow R$ and $G_{b}: H \times R^{+} \longrightarrow R$ by $G_{a}(\theta, L)=L \int_{I} \cos \theta-a$ and $G_{b}(\theta, L)=$ $L \int_{I} \sin \theta-b$. Assume the curve have initial point at the origin, and write $x(s)=$ $L \int_{0}^{s} \cos (t) d t$ and $y(s)=L \int_{0}^{s} \sin (t) d t$. If it is assumed that $x(1)-x(0)=a$ and $y(1)-y(0)=b$, then the derivatives are

$$
\begin{gathered}
D G_{a}(\theta, L)\left(v_{\theta}, v_{L}\right)=\int_{I} \dot{v}_{\theta}(y(s)-b) d s-v_{\theta}(0) b+a v_{L} / L, \\
D G_{b}(\theta, L)\left(v_{\theta}, v_{L}\right)=-\int_{I} \dot{v}_{\theta}(x(s)-a) d s+v_{\theta}(0) a+b v_{L} / L
\end{gathered}
$$

and the gradients are

$$
\begin{gathered}
\nabla G_{a}(\theta, L)=\left(\int_{0}^{s} y(t) d t-b s-b, a / L\right), \\
\nabla G_{b}(\theta, L)=\left(-\int_{0}^{s} x(t) d t+a s+a, b / L\right) .
\end{gathered}
$$

Let $\Omega$ represent all curves with gap $(a, b)$ and write $J_{\Omega}^{\nu}$ for the restriction of $J^{\nu}$ to $\Omega$. Now find the real multipliers $\lambda_{a}, \lambda_{b}$ so that

$$
\nabla J^{\nu}=\nabla J_{\Omega}^{\nu}+\lambda_{a} \nabla G_{a}+\lambda_{b} \nabla G_{b} .
$$

The fact that $\nabla J_{\Omega}^{\nu}$ is tangent to $\Omega$ implies that $D G_{a}\left(\nabla J_{\Omega}^{\nu}\right)=0$ and $D G_{b}\left(\nabla J_{\Omega}^{\nu}\right)=0$. This linear system can be expressed as a matrix equation:

$$
\begin{gathered}
M=\left[\begin{array}{cc}
\int_{I}(y-b)^{2}+b^{2}+a^{2} / L^{2} & -\int_{I}(x-a)(y-b)-a b+a b / L^{2} \\
-\int_{I}(x-a)(y-b)-a b+a b / L^{2} & \int_{I}(x-a)^{2}+a^{2}+b^{2} / L^{2},
\end{array}\right], \\
M\left[\begin{array}{c}
\lambda_{a} \\
\lambda_{b}
\end{array}\right]=\left[\begin{array}{c}
\frac{a}{L}\left(\nu-\frac{1}{2 L^{2}} \int_{I} \dot{\theta}^{2}\right)+\int_{I} k(y-b) \\
\frac{b}{L}\left(\nu-\frac{1}{2 L^{2}} \int_{I} \dot{\theta}^{2}\right)-\int_{I} k(x-a)
\end{array}\right] .
\end{gathered}
$$

For closed curves with $a=b=0$ one recognizes the inertia tensor and the Steiner curvature centroid discussed in [6]. The hope for explicit examples of flows is dashed by the fact that the length part, $\nu-\frac{1}{2 L^{2}} \int_{I} \dot{\theta}^{2}$, of $\nabla J^{\nu}$ is still present in the flow equation. The idea of restricting to curves with a vanishing Steiner curvature centroid is not productive here. This idea yielded a family of explicit flows in the length constrained case; see [6].

3.2 The semi-circle. The matrix equation in this case is

$$
\left[\begin{array}{cc}
\frac{1}{8}+\frac{4}{\pi^{2}} & -\frac{1}{2 \pi} \\
-\frac{1}{2 \pi} & \frac{11}{8}
\end{array}\right]\left[\begin{array}{c}
\lambda_{a} \\
\lambda_{b}
\end{array}\right]=\left[\begin{array}{c}
-\frac{6}{\pi} \\
1
\end{array}\right] .
$$

The gradients are given by

$$
\begin{gathered}
\nabla J^{\nu}=(2 s,-2), \\
\nabla G_{a}=\left(\frac{1}{2 \pi}(\cos (\pi s)-1), \frac{2}{\pi}\right), \\
\nabla G_{b}=\left(\frac{1}{2 \pi} \sin (\pi s)+\frac{s}{2}+1,0\right) .
\end{gathered}
$$

It follows that when the first component of $\nabla J_{\Omega}^{\nu}$ is evaluated at $s=0$ and $s=1$ one gets $-\lambda_{b}$ and $2+\lambda_{a} / \pi-3 \lambda_{b} / 2$ respectively. The first component corresponds to the 


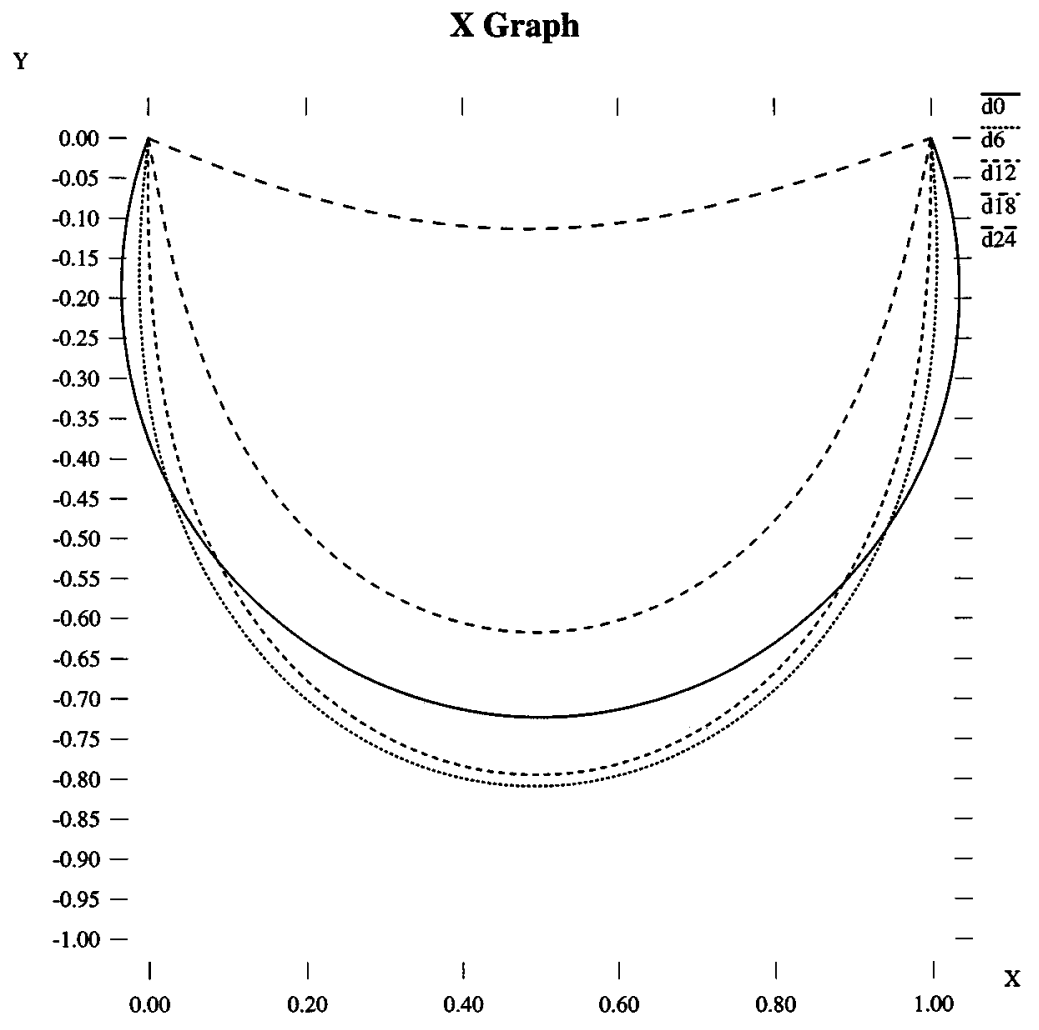

Figure 6

tangent angle of the curve. If the reflectional symmetry is to be preserved it must be that $-\lambda_{b}$ is the negative of $2+\lambda_{a} / \pi-3 \lambda_{b} / 2$, or equivalently $5 \lambda_{b} / 2-\lambda_{a} / \pi=2$. Proceed by replacing $\lambda_{a}$ and $\lambda_{b}$ in

$$
\frac{1}{2 \pi}\left[\begin{array}{ll}
-2 & 5 \pi
\end{array}\right]\left[\begin{array}{c}
\lambda_{a} \\
\lambda_{b}
\end{array}\right]
$$

using the matrix equation. The result may be written as

$$
\frac{\frac{20}{64}+\frac{41}{4 \pi^{2}}}{\frac{11}{64}+\frac{21}{4 \pi^{2}}},
$$

where the denominator is the determinant of $M$. The expression is not equal to 2 and therefore the reflectional symmetry is not preserved.

3.3 Length variation along trajectories. An interesting numerical observation concerns the way the length changes during the curve straightening flow. By considering more general arcs of a circle it is seen that for sufficiently long circular arcs the flow increases the length without bounds. There is a circular arc where the length component of the gradient vanishes but the tangent angle component is not zero. Ultimately, this arc converges to the straight line segment between the endpoints. It is surprising that initially the length actually increases. This behavior is seen even better for a slightly different circular arc; see Figure 6 . 


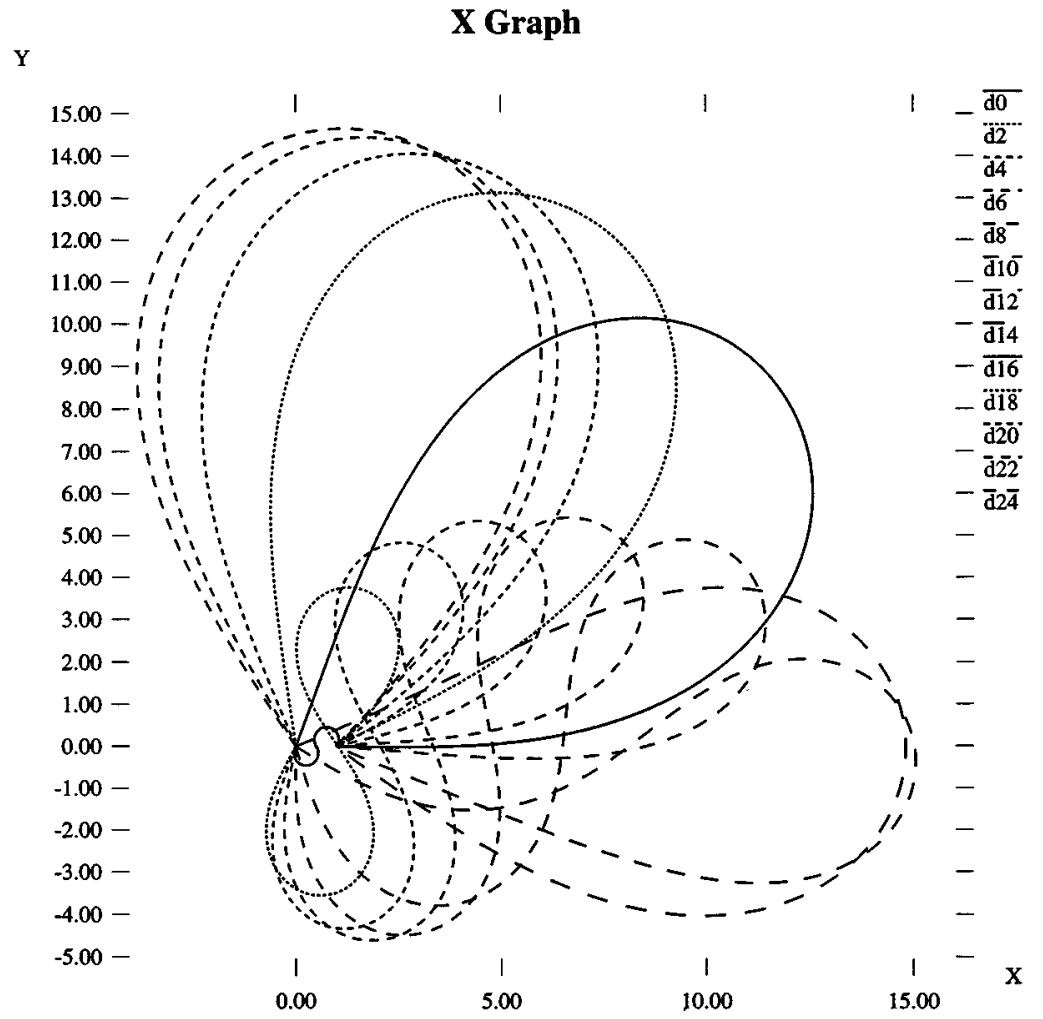

FiguRE 7

3.4 Preserved symmetry. Symmetry is not preserved in general. For instance, when $\nu=0$ a circle tends to half of the Euler figure eight. The first example, given by Figure 7, illustrates how rotational symmetry about a point is not preserved. As in the case of the semi-circle an explicit computation shows that the gradient does not preserve the symmetry. For closed curves $a=b=0$ and the length dependent terms drop out of the matrix equation. The second example is given by Figure 8. In this case the inertia tensor is diagonal. This time an explicit computation shows that the gradient evaluated at the initial curve preserves the symmetry. In the case of closed curves of fixed length with smooth tangent angles the curve straightening flow is known explicitly when the curve is symmetric through a point and the rotation number is equal to 1 ; see [6]. In this case it is shown that the flow preserves the symmetry for all flow-times.

Finally, a numerical simulation suggests that the critical points at infinity which consist of two halves or more of the Euler figure eight are all 'unstable'. Consequently the flow towards 'infinity' resembles walking along a ridge. It serves as a good test of the numerical implementation. The accumulated errors eventually force the flow off the ridge, and the later this happens the better the algorithm.

\subsection{Divergent trajectories and critical points at infinity.}

3.5.1 Indefinite extension of all negative gradient trajectories. The idea of the following proof can be found in [11]. It is included here since we wish to point out 
Y

\section{Graph}

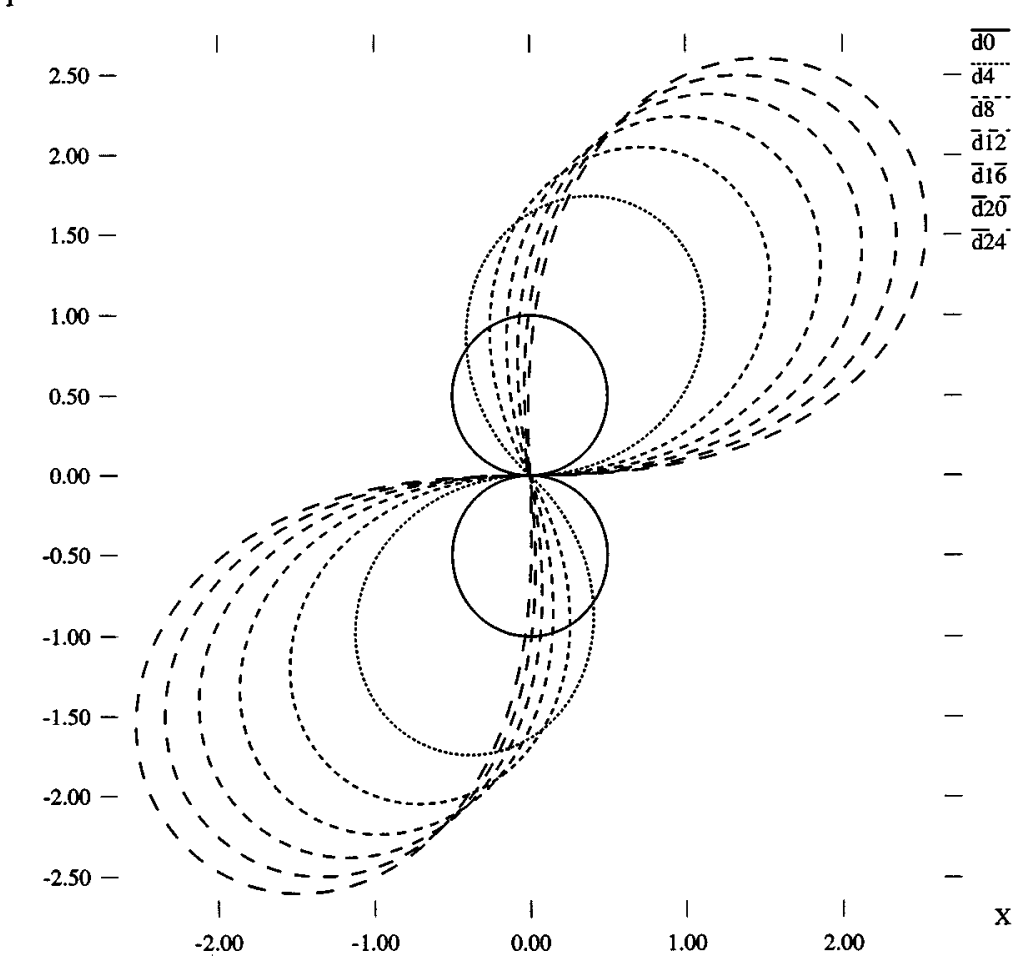

FiguRE 8

that there is no need to assume the Palais-Smale condition for the argument to work. Note that the nonlinear functional must be bounded from below.

Proposition 3.1. Suppose $F: X \longrightarrow[0, \infty)$ is smooth and $X$ is a complete Hilbert manifold. Then all negative gradient trajectories can be extended indefinitely and the gradient tends to zero along the trajectory.

Proof. Suppose $x \in X$ is given and let $\sigma:[0, a) \longrightarrow X$ be such that $\sigma(0)=x$ and $\sigma^{\prime}(t)=-\nabla F(\sigma(t))$. Assume that $a$ is maximal with this property.

Suppose that $a<\infty$ and that $\sigma(t)$ has a limit point $x_{a} \in X$ as $t$ tends to $a$ from below. From standard theory it follows that there is a curve $\bar{\sigma}$ defined in an open interval about 0 with $\bar{\sigma}(0)=x_{a}$ and $\bar{\sigma}^{\prime}(t)=-\nabla F(\bar{\sigma}(t))$ for all $t$ in the interval. To get a contradiction to the maximality of $a$ it suffices to join $\sigma$ and $\bar{\sigma}$ in the obvious fashion.

If the length of $\sigma$ is finite, it is not hard to show that $\sigma([0, a))$ is totally bounded. Since $X$ is assumed to be complete, this implies there exists a limit point. Combine this with the previous and conclude that either $a=\infty$ or the length of $\sigma$ is infinite.

By assumption $F(\sigma(t)) \geq 0$. Since $F(\sigma(t))-F(x)=-\int_{0}^{t}\|\nabla F(\sigma(s))\|^{2} d s$, it follows that $\int_{0}^{t}\|\nabla F(\sigma(s))\|^{2} d s \leq F(x)$. This combined with

$$
\int_{0}^{a}\left|\sigma^{\prime}(s)\right| d s=\int_{0}^{a}\|\nabla F(\sigma(s))\| d s \leq \sqrt{a}\left(\int_{0}^{a}\|\nabla F(\sigma(s))\|^{2} d s\right)^{\frac{1}{2}}
$$


implies that the length of $\sigma$ is finite if $a$ is finite. The only way out is to accept that $a=\infty$. Finally, if $\|\nabla F(\sigma(s))\|$ is bounded away from zero, then the first inequality leads to a contradiction since as $t$ tends to infinity $\int_{0}^{t}\|\nabla F(\sigma(s))\|^{2} d s$ is unbounded.

3.5.2 Verification of the Palais-Smale condition when the length is bounded. In the next proposition some of the techniques developed in [4] are adapted to the present context. There are four differences here: the length is variable, the directions at the endpoints are not fixed, the curves are not closed and the curves are planar. These differences affect the reasoning in several ways. Since the curves are planar the indicatrix is identified with a single real-valued function. In [4] the indicatrix is a map into $S^{2}$, and thus there is a need to use the covariant derivative. Due to the variable length there is an extra term in the inner product. When the curves are not closed a crucial part of the argument actually simplifies.

Let $H^{0}=L^{2}[I]$ with inner product $\langle v, w\rangle_{0}=\int_{I} v w$, and let $H^{1}$ be the set of absolutely continuous functions with derivatives in $H^{0}$ and inner product $\langle v, w\rangle_{1}=$ $v(0) w(0)+\langle\dot{v}, \dot{w}\rangle_{0}$. The ambient domain in this context is $H=\left\{\theta \in H^{1} \mid \theta(0) \in\right.$ $R \bmod 2 \pi\}$. Let $R^{+}$be the positive reals and consider the space $H \times R^{+}$. The tangent spaces are identified with $H \times R$ with inner product $\left\langle\left(v_{\theta}, v_{L}\right),\left(w_{\theta}, w_{L}\right)\right\rangle_{+}=$ $\left\langle v_{\theta}, w_{\theta}\right\rangle_{1}+v_{L} w_{L}$. Let $J: H \times R^{+} \longrightarrow R$ be given by $J(\theta, L)=\frac{1}{2 L} \int_{I} \dot{\theta}^{2}$. Given a complex number $g$, define $\Lambda: H \times R^{+} \longrightarrow C$ by $\Lambda(\theta, L)=L \int_{I} e^{i \theta}-g$ and assume $g \neq 0$.

Proposition 3.2. Suppose $p_{n}=\left(\theta_{n}, L_{n}\right)$ is a sequence in $\Omega$ such that both $J_{\Omega}\left(p_{n}\right)$ and $L_{n}$ are bounded above. Assume that $\nabla J_{\Omega}\left(p_{n}\right)$ converges in $H \times R$ but not necessarily to zero. Then there is a subsequence of $p_{n}$ which converges in $\Omega$. In particular, it follows that if the length is bounded then the Palais-Smale condition is satisfied.

Proof. First we prove that, for some subsequence, $\nabla J\left(p_{n_{k}}\right)$ converges. Given $p \in \Omega$, there is a unique complex number $\lambda$ such that

$$
\nabla J(p)=\nabla J_{\Omega}(p)+D \Lambda(p)^{T}(\lambda) .
$$

If $\lambda_{n}$ is bounded and $D \Lambda\left(p_{n}\right)^{T} u_{n}$ converges in $H$ for some unit vectors $u_{n}$, then by the above equation it must be that, for some subsequence $p_{n_{k}}, \nabla J\left(p_{n_{k}}\right)$ converges in $H$.

To bound $\lambda_{n}$, observe that

$$
\begin{aligned}
& \nabla J_{\Omega}(\theta, L) \\
& \quad=\left(\frac{1}{L}(\theta(s)-\theta(0))-\left\langle-i L \int_{0}^{s} \int_{0}^{t} e^{i \theta}+i(s+1) g, \lambda\right\rangle,-\frac{1}{2 L^{2}} \int_{I} \dot{\theta}^{2}-\left\langle\frac{g}{L}, \lambda\right\rangle\right)
\end{aligned}
$$

Consider the second component and recall that it is bounded by assumption. The first term is $-J(\theta, L) / L$, which is bounded as well. The only way $\lambda_{n}$ can be unbounded is if $\lambda_{n} /\left|\lambda_{n}\right|$ tends to the perpendicular direction $i g /|g|$. To proceed, consider the first component. It is also bounded by assumption. Let $s=0$ and get $\left\langle i g, \lambda_{n}\right\rangle$. This leads to a contradiction if $\lambda_{n}$ is unbounded. Hence $\lambda_{n}$ is bounded.

Next prove that, for some subsequence, $D \Lambda\left(p_{n}\right)^{T} u_{n}$ converges in $H$. Since $L_{n}$ is bounded it suffices to look at

$$
\beta_{n}(s)=\left\langle-i L_{n} \int_{0}^{s} \int_{0}^{t} e^{i \theta_{n}}+i(s+1) g, u_{n}\right\rangle,
$$




$$
\begin{gathered}
\dot{\beta}_{n}(s)=\left\langle-i L_{n} \int_{0}^{s} e^{i \theta_{n}}+i g, u_{n}\right\rangle, \\
\left\|\dot{\beta_{n}}(s)\right\|_{1}^{2}=\left\langle i g, u_{n}\right\rangle^{2}+\int_{I}\left\langle-i L_{n} e^{i \theta_{n}}, u_{n}\right\rangle^{2} .
\end{gathered}
$$

The bound on $L_{n}$ shows that $L_{n} e^{i \theta_{n}}$ is in a fixed disc for all $n$, and as a consequence the integral term is bounded. It follows that $\dot{\beta}_{n}$ is bounded in $H^{1}$. By an embedding theorem some subsequence $\dot{\beta}_{n_{k}}$ converges in $C^{0}$ as well as $H^{0}$. Hence $\beta_{n_{k}}$ converges in $H^{1}$.

To finish the proof, assume that the sequence is such that $\nabla J\left(p_{n}\right)$ converges in $H \times R$. Here

$$
\nabla J\left(p_{n}\right)=\nabla J\left(\theta_{n}, L_{n}\right)=\left(\frac{1}{L_{n}}\left(\theta_{n}(s)-\theta_{n}(0)\right),-\frac{1}{2 L_{n}^{2}} \int_{I} \dot{\theta}_{n}^{2}\right)
$$

Put $\alpha_{n}(s)=\frac{1}{L_{n}}\left(\theta_{n}(s)-\theta_{n}(0)\right)$ and let $\hat{\alpha}$ be in $H$ so that $\alpha_{n}$ converges to $\hat{\alpha}$ in $H$, and $\theta_{n}(s)-\theta_{n}(0)=L_{n} \alpha_{n}(s)$. Use the bound on $L_{n}$ and the fact $\theta_{n}(0) \in R \bmod 2 \pi$ to extract yet another subsequence $\left(\theta_{n_{k}}, L_{n_{k}}\right)$ which converges in $H$. Specifically, let

$$
\theta_{n_{k}}(s)=L_{n_{k}} \alpha_{n_{k}}(s)+\theta_{n_{k}}(0),
$$

where $L_{n_{k}}$ converges to $\hat{L}$ and $\theta_{n_{k}}(0)$ converges to $\hat{\theta}_{0}$. Look at

$$
\left\|\left(\theta_{n_{k}}-\left(\hat{L} \hat{\alpha}+\hat{\theta}_{0}\right), L_{n_{k}}-\hat{L}\right)\right\|_{+}^{2}=\left(\theta_{n_{k}}(0)-\hat{\theta}_{0}\right)^{2}+\int_{I}\left(\dot{\theta}_{n_{k}}-\hat{L} \dot{\hat{\alpha}}\right)^{2}+\left(L_{n_{k}}-\hat{L}\right)^{2} .
$$

The first term and the third term both tend to zero. The square in the middle term is expands to three more terms. The two square terms are immediately seen to converge to zero. The mixed product term is bounded by the Cauchy-Schwarz inequality, and the bound converges to zero. Finally, since $\Lambda\left(\theta_{n}, L_{n}\right)=0$ for all $n$ and $\Lambda$ is continuous on $C^{0} \times R^{+}$, it must be that $\Lambda(\hat{\theta}, \hat{L})=0$, so the limit in $H$ is also in $\Omega$.

\subsubsection{Existence of divergent trajectories.}

Theorem 3.3. Suppose $\nu=0$, and consider the negative gradient flow associated with $J_{\Omega}^{0}: \Omega \longrightarrow R$, where $\Omega$ is the space of indicatrices corresponding to curves of variable length, whose endpoints are separated by the vector $g$, and $J_{\Omega}^{0}(\theta, L)=$ $\frac{1}{2 L} \int_{I} \dot{\theta}^{2}$. There exist initial curves and corresponding negative gradient trajectories such that the lengths of the curves along the trajectory tend to infinity. If $g=0$, the length of the curves along the trajectory tend to infinity for all initial curves. If $g \neq 0$, then all initial curves of length greater than the fixed length $\left(2 K^{2} / \pi\right)|g| \approx$ $2.18844|g|)$ of the non-geodesic critical points, and with functional value less than the minimal non-geodesic value $\pi^{2} / K^{2}|g| \approx 2.87108 /|g|$, have trajectories along which the length tends to infinity.

Proof. Begin with closed curves so that $g=0$. First consider only curves $(\theta, L) \in$ $H \times R^{+}$of length $L=1$ such that $\int_{I} e^{i \theta}=0$. The minimizer in this class is given by one half of the Euler figure eight; see Figure 4. It follows that the functional value $\int_{I} \dot{\theta}^{2}$ is bounded below by a positive value. Curves of arbitrary length $L$ satisfy $L \int_{I} e^{i \theta}=0$, so again $\int_{I} e^{i \theta}=0$. The length component of the gradient is given by $\frac{1}{2 L^{2}} \int_{I} \dot{\theta}^{2}+\lambda_{a} a / L+\lambda_{b} b / L$, and by Proposition 3.1 it tends to zero along 
a trajectory. Now $g=0$, so $a=b=0$, and since the integral is bounded below by a positive number, it must be that the length tends to infinity.

Next consider nonclosed curves so that $g \neq 0$. Recall that, except for the straight line segment, all the critical points have the same length $\left(L_{C}\right)$; see Figure 1. Note that many critical points converge to the straight line segment; see Figure 5. Suppose the initial curve has length $L_{0}>L_{C}$ and assume the functional value is smaller than the minimizing value among the non-geodesic critical points. An explicit example is given by a curve consisting of two parallel straight line segments of the same length, connected by a semi-circle. For sufficiently long line segments the above conditions are satisfied.

Consider the negative gradient trajectory $\sigma$, starting at an initial curve of the above kind. Suppose the length is bounded along the trajectory. Let $\sigma(n)=$ $\left(\theta_{n}, L_{n}\right)$ be a sequence along the trajectory. Since the gradient and the functional are independent of $\theta_{n}(0)$, it is possible to create another sequence $\left(\widetilde{\theta}_{n}, L_{n}\right)$ with $\widetilde{\theta}_{n}(0)$ in $R \bmod 2 \pi$, and now Proposition 3.2 is applicable. By Proposition 3.2 the Palais-Smale condition is satisfied. The lengths $L$ of the curves along the trajectory must be bigger than $L_{C}$, since otherwise the minimality of the functional among the critical points is contradicted. It is here important that the functional is decreasing along the trajectory and it starts at a lower value. Since the P-S condition is satisfied and since the gradient on $\left(\widetilde{\theta}_{n_{k}}, L_{n_{k}}\right)$ tends to zero, there must be another critical point, but there are no other critical points. The theorem follows as the only remaining possibility.

3.5.4 Examples. Figures 9-14 give three examples of initial curves that diverge to infinity. In Figures 9 and 10 the initial curve is a one and a half wrapped circle. In Figures 11 and 12 the initial curve is a straight line segment with a small circular loop in the middle. In Figures 13 and 14 the initial curve is a straight line segment which in the middle has a small figure eight consisting of two circles. The examples also illustrate how half of the Euler figure eight acts like a critical point at infinity.

3.6 Dragging trajectories to infinity. As mentioned earlier, decreasing $\nu$ to zero causes 'half' of the critical points to migrate to infinity. Examples indicate that as this takes place these critical points attract trajectories, and they do not 'eat up' these trajectories on their way to infinity. In Figure 15 the initial curve d0 is part of a circle. As the parameter $\nu$ is decreased by a factor of two starting at $\nu=0.02$, there is always a critical point to which the gradient trajectory converges because the Palais-Smale condition is satisfied. The flow is followed all the way to the critical point, so d1 corresponds to the critical point in the case of $\nu=0.02$ and $\mathrm{d} 2$ corresponds to the critical point in the case of $\nu=0.01$, and so on and so forth. Note how the length of the curve tends to infinity as $\nu$ tends to zero.

The author plans to deal with the subject of constrained endpoint directions in greater detail in a subsequent paper. Of particular interest is the case of smooth closed curves in the standard round sphere and the hyperbolic disc. As mentioned in the introduction, there are no migrating critical points in the hyperbolic case. The spherical case is less understood, but there is still hope that there are no migrating critical points in this case as well. In the plane the very long curves are not 'close' to any critical points, so trajectories have no place to go. This is not the case in the sphere, since there are arbitrarily long geodesics. This is a very 'bullish' sign for the convergence of the trajectories in spite of a failing Palais-Smale condition. 


\section{Graph}
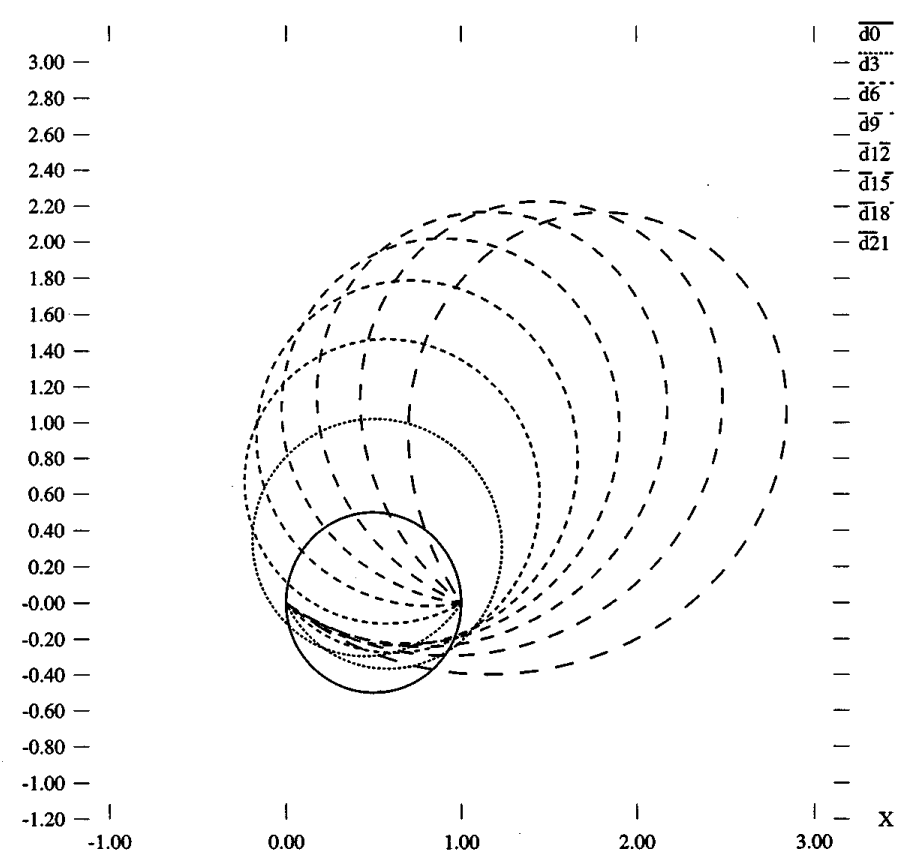

Figure 9

Y

3.00-
$2.50-$
$2.00-$
$1.50-$
$1.00-$
$0.50-$
$0.00-$
$-0.50-$
$-1.00-$
$-1.50-$
$-2.00-$
$-2.50-$
$-3.00-$
$-3.50-$
$-4.00-$
$-4.50-$
$-5.00-$
$-5.50-$
$-6.00-$
$-6.50-$
$-7.00-$
-4.00

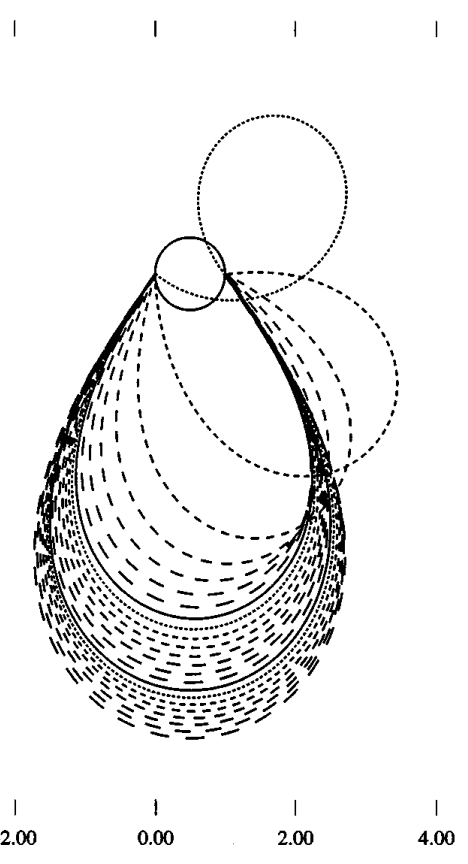

$$
\begin{aligned}
& 1 \frac{\mathrm{do}}{\mathrm{d}} \\
& \begin{array}{l}
-\mathrm{d} 1 \\
-\mathrm{d}^{-}-
\end{array} \\
& -\mathrm{d} 3 \text {. } \\
& -\frac{\bar{d} 4}{\mathrm{~d} 5} \\
& -\frac{\mathrm{d}}{\mathrm{d} 6} \text {. } \\
& -\frac{\bar{d} 6}{d 7} \\
& -\overline{d 8} \\
& -\frac{\mathrm{d} 9}{\mathrm{~d} i 0} \\
& -\frac{\mathrm{d}}{\mathrm{i}} \mathrm{i} \\
& -\overline{\mathrm{d}} \overline{2} \\
& -\bar{d} 1 \overline{3} \\
& -\overline{\mathrm{d} 14} \\
& \begin{array}{r}
-\overline{\mathrm{d} 14} \\
-\overline{\mathrm{d} 15} \\
\hline
\end{array} \\
& \overline{\mathrm{d} 16} \\
& \begin{array}{l}
-\mathrm{di} 7 \\
-\mathrm{d} 18
\end{array} \\
& \begin{array}{l}
-\mathrm{d} 18 \\
-\overline{\mathrm{d}} \overline{10}
\end{array} \\
& \begin{array}{l}
-\overline{\mathrm{d}} \overline{9} \\
-\overline{\mathrm{d}} 2 \overline{0}
\end{array} \\
& -\overline{\mathrm{d}} 2 \overline{1} \\
& -\frac{\mathrm{d} 22}{\mathrm{~d} 23} \\
& - \\
& \text { - }
\end{aligned}
$$

Figure 10 
Y

\section{Graph}
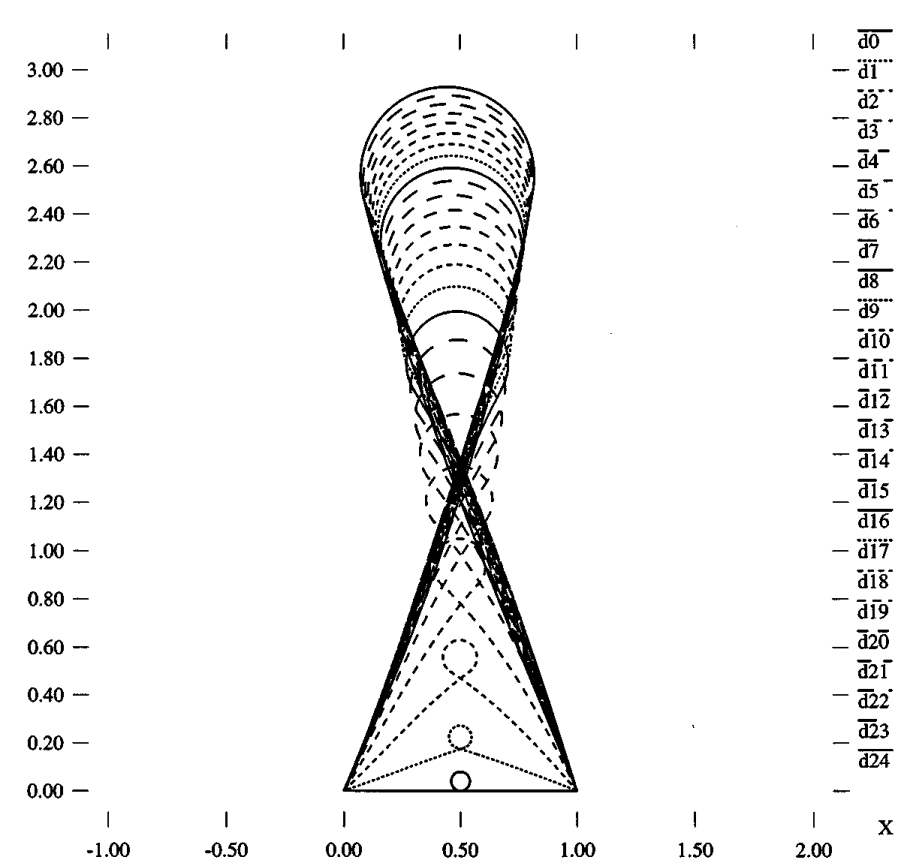

FIGURE 11

$\mathrm{Y}$

X Graph

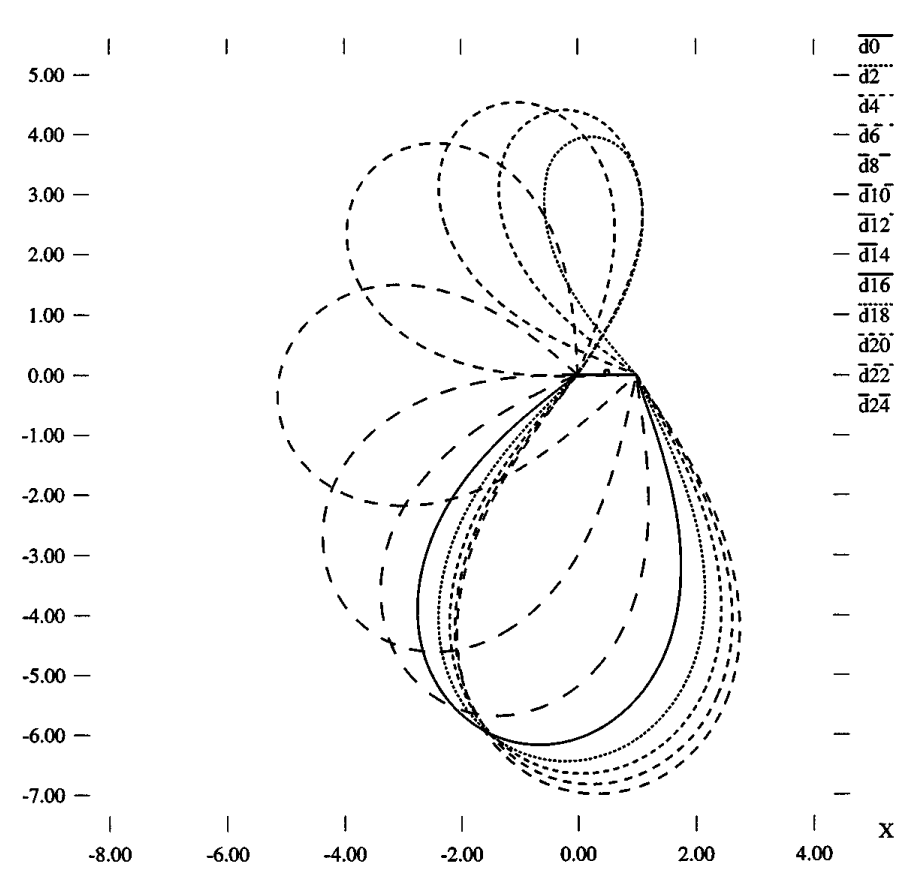

FIGURE 12 


\section{Graph}

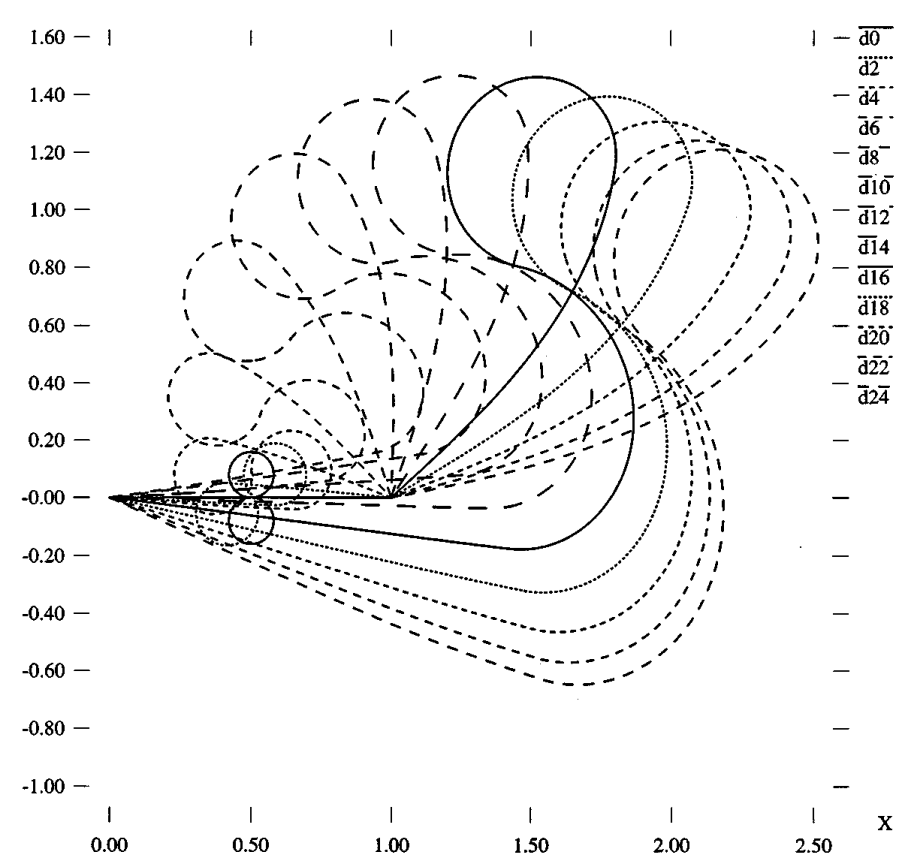

Figure 13

X Graph

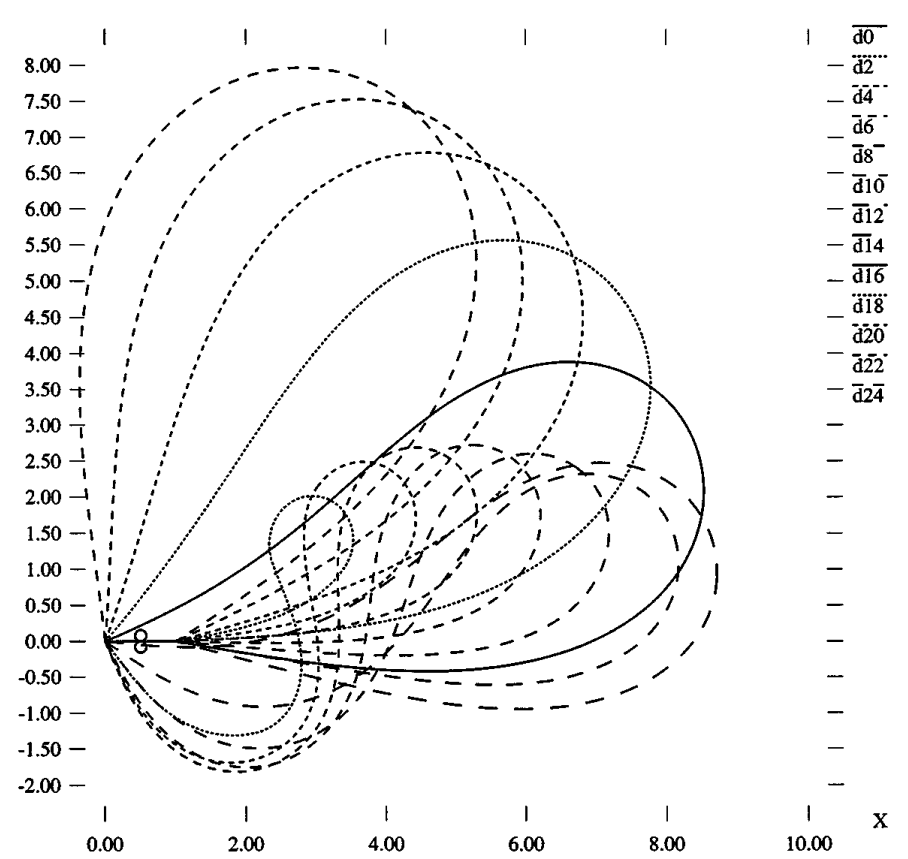

Figure 14 


\section{Graph}

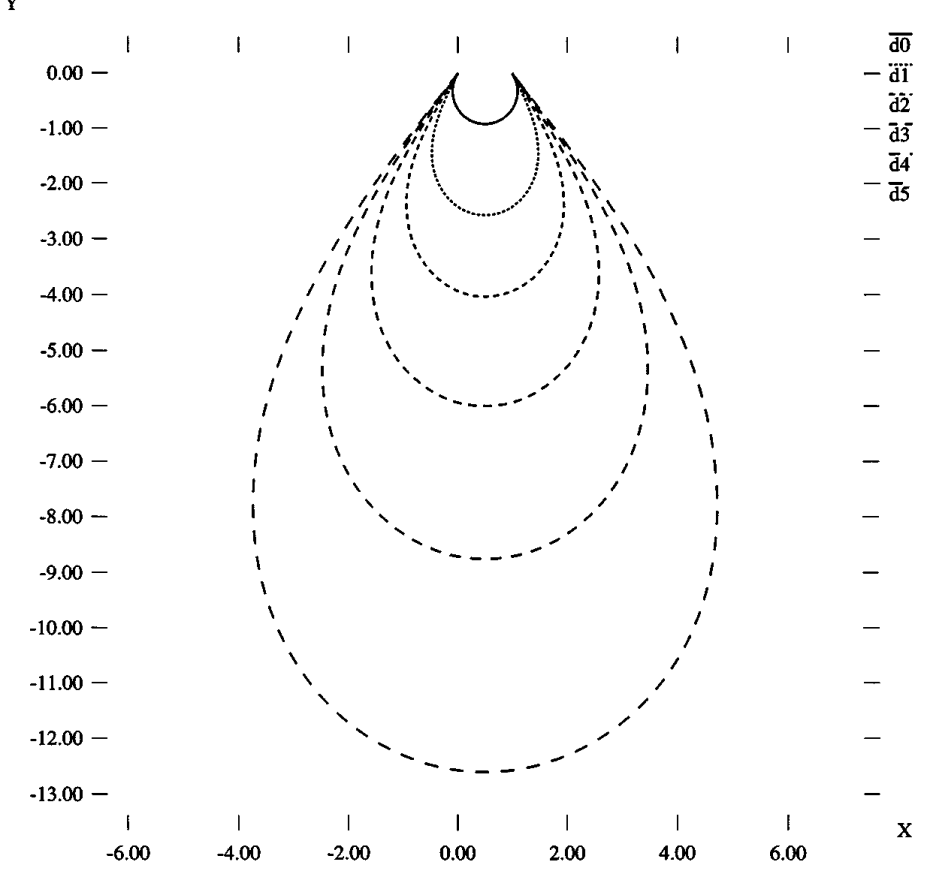

FiguRE 15

\section{REFERENCES}

[1] Franks, J., Geodesics on $S^{2}$ and periodic points of annulus homeomorphisms, Invent. Math. 108 (1992), 403-418. MR 93f:58192

[2] Klingenberg, W, Lectures on Closed Geodesics, Springer-Verlag, 1978. MR 57:17563

[3] Langer, J. and Singer, D. A., The total squared curvature of closed curves, J. Diff. Geo. 20 (1984), 1-22. MR 86i:58030

[4] Langer, J. and Singer, D. A., Curve-straightening and a minimax argument for closed elastic curves, Topology 24 (1985), 75-88. MR 86j:58023

[5] Langer, J. and Singer, D. A., Curve-straightening in Riemannian manifolds, Ann. Global Anal. Geom. 5 (1987), 133-150. MR 89i:58025

[6] Linnér, A., Some properties of the curve straightening flow in the plane, Trans. Amer. Math. Soc. 314 (1989), 605-617. MR 90g:58022

[7] Linnér, A., Steepest descent used as a tool to find critical points of $\int k^{2}$ defined on curves in the plane with arbitrary types of boundary conditions, Geometric Analysis and Computer Graphics, MSRI Publications, Vol. 17, Springer-Verlag, 1991, 127-138. MR 91j:58041

[8] Linnér, A., Existence of free non-closed Euler-Bernoulli elastica, Nonlinear Analysis 21 (1993), 575-593. MR 94m:58047

[9] Linnér, A., Unified representations of nonlinear splines, J. Approx. Theory 84, No. 3 (1996), 315-350. MR 97f:41012

[10] Palais, R. S., Morse theory on Hilbert manifolds, Topology 2 (1963), 299-340. MR 28:1633

[11] Palais, R. S. and Terng, C., Critical Point Theory and Submanifold Geometry, Lect. Notes in Math. 1353, Springer-Verlag, 1988. MR 90c:53143

[12] Steinberg, D. H., Thesis: Elastic curves in hyperbolic space, Case Western Reserve University (1995).

Department of Mathematical Sciences, Northern Illinois University, DeKalb, IlliNOIS 60115

E-mail address: alinner@math.niu.edu 